\title{
The Effect of Uniform Magnetic Field on Pressurized FG Cylindrical and Spherical Vessels
}

\author{
Anwr Temo $(\mathbb{B}$, Durmuş Yarımpabuç*i] \\ Department of Mathematics, Osmaniye Korkut Ata University, Osmaniye, Turkey
}

\begin{abstract}
The stress analysis of functionally graded thick hollow cylindrical and spherical pressure vessels under the effect of uniform magnetic field are examined. These pressure vessels are designed in such a way that the material properties and magnetic permeability are exponentially graded in radial direction, provided that the inner surface is pure metal and the outer surface is pure ceramic. The differential equations with variable coefficients obtained under these conditions are handled by both Complementary Functions Method and Pseudospectral Chebyshev Method. Benchmark solutions available in the literature for some special cases are used to confirm the results. The effects of different mixture and uniform magnetic field on stresses and displacement distribution are shown in graphical form.
\end{abstract}

Keyword: Functionally Graded Materials, Uniform magnetic field, Stress analysis, Pseudospectral Chebyshev Method, Complementary Functions Method

\section{INTRODUCTION}

Recently, due to the need for both high strength and heat resistant materials, scientists began to work on different materials structures. The idea of functionally grading the thermomechanical properties of particulate composites was first introduced and developed by a group of materials scientists working on materials in Japan [1,2]. In this context, the functionally graded materials (FGMs) [3] are obtained by forming different types of materials according to a certain rule. These materials are generally considered to be a model that varies continuously from one surface to another. They can be produced in a macroscopic way, both in resistance to high temperature environments and in optimizing the stress and displacement of the material. FGMs can be used in many areas, such as in nuclear field, space field, medical field, energy field, etc.

The stress analysis of the functional graded thick hollow cylinder and sphere have been investigated deeply in the literature. Horgan and Chan [4] investigated the effects of functionally graded circular cylinders and disks on displacement and stresses under uniform internal and external pressure. The exact analysis of the functionally graded cylindrical and spherical vessels under the plane-strain assumption with radially graded elastic modulus are readily available $[5,6]$. Using the infinitesimal theory of elasticity, Yarımpabuç et.al.
[7-9] investigated the effect of the both Young's modulus and Poisson's ratio that are graded radially on the FG vessels numerically. There are many other studies on these subjects, e.g., [10-20], where an additional reference can also be found.

Dai et.al. [21], presented the magnetoelastic behavior of the functionally graded cylindrical and spherical vessels under the effect of uniform magnetic field with radially graded elastic modulus and magnetic permeability by using the infinitesimal theory of magnetoelasticity. Dai et.al. [22], presented the exact analysis of the functionally graded cylindrical and spherical structures, subjected to mechanical loading and electric excitation with radially graded mechanical, piezoelectric and dielectric properties by using the axisymmetric plain strain assumption.

In this study, the stress analysis of functionally graded thick hollow cylindrical and spherical vessels subjected to an internal pressure under the effect of uniform magnetic field are numerically handled by both the Complementary Function Method (CFM), [23, 24] and the Pseudospectral Chebyshev Method (PCM), $[25,26]$. It is assumed that the pressure vessels are composed of ceramic and metal, alternating exponentially through the radial direction. In addition, magnetic permeability is also changed in the same way. These conditions produce a linear ordinary differential equation that 
may not be solved analytically with conventional methods except for some simple grading functions. Therefore, numerical solution is essential. The results of stresses and displacement distributions are discussed for two different mixture with two different approach. The mixture of the materials is chosen in such a way that the Poisson's ratio in one does not change, while in the other there is a slight difference. The results obtained from the study of Dai et.al.[21] are used for validation purposes. The stress and displacement distributions under the effects of two different mixture materials and uniform magnetic field that are both grading exponentially are shown in graphical form.

\section{THE FORMULATION OF PRESSURE VESSELS}

The stress analysis of functionally graded thick cylindrical and spherical vessels subjected to an internal pressure under the effect of uniform magnetic field are discussed numerically. The inner and outer radius of the thick-hollow bodies are taken as and respectively. The cross-section of the functionally graded body is shown in Figure 1. It is assumed that these bodies are made from a mixture of metal and ceramic and the all material properties and magnetic permeability are graded exponentially in radial direction as:

$$
\begin{aligned}
& E(r)=E_{i} e^{\beta(r-a)} \\
& v(r)=v_{i} e^{\gamma(r-a)} \\
& \mu(r)=\mu_{i} e^{w(r-a)}
\end{aligned}
$$

Here $E, v, \mu$ and $(E i, v i, \mu i)$ are the modulus of elasticity, Poisson's ratio, magnetic permeability and material constants in the inner boundary, respectively. The constants $(\beta, \gamma, w)$ indicate the inhomogeneity parameters and can be calculated from the following relations:

$$
\begin{aligned}
& \beta=\frac{1}{b-a} \ln \left(\frac{E_{0}}{E_{i}}\right) \\
& \gamma=\frac{1}{b-a} \ln \left(\frac{v_{0}}{v_{i}}\right) \\
& \omega=\frac{1}{b-a} \ln \left(\frac{\mu_{0}}{\mu_{i}}\right)
\end{aligned}
$$

where $\left(E_{0}, v_{0}, \mu_{0}\right)$ are the material constants in the outer boundary. The subscripts " $i$ " and "0" show the material properties of the inner and outer boundary. It is supposed that the thick hollow bodies have a pressure only on its inner surface, so the boundary conditions for the radial stress are:

$$
\sigma(r=a)=-P, \sigma(r=b)=0
$$

Here, $\sigma_{r}$ is radial stress of the bodies and $P$ is the pressure in the inner surface.

\section{CYLINDRICAL VESSELS}

Functionally graded infinitely long hollow cylinder made from a mixture of metal and ceramic under the effect of a uniform magnetic field $\vec{H}\left(0,0, H_{z}\right)$ is considered. Under the axisymmetric plain strain assumption, the stress-strain and strain-displacement equations in cylindrical coordinate $(r, \theta, z)$ can be written as follow:

$$
\begin{aligned}
& \sigma_{r}=C_{11}(r) \varepsilon_{r}+C_{12}(r) \varepsilon_{\theta} \\
& \sigma_{\theta}=C_{11}(r) \varepsilon_{\theta}+C_{12}(r) \varepsilon_{r}
\end{aligned}
$$

and

$$
\varepsilon_{r}=\frac{d u}{d r}, \quad \varepsilon_{\theta}=\frac{u}{r}
$$

where,

$$
\begin{aligned}
& C_{11}(r)=\left(\frac{E_{i} e^{\beta(r-a)}\left(1-v_{i} e^{\gamma(r-a)}\right)}{\left(1+v_{i} e^{\gamma(r-a)}\right)\left(1-2 v_{i} e^{\gamma(r-a)}\right)}\right) \\
& C_{12}(r)=\left(\frac{E_{i} e^{\beta(r-a)} v_{i} e^{\gamma(r-a)}}{\left(1+v_{i} e^{\gamma(r-a)}\right)\left(1-2 v_{i} e^{\gamma(r-a)}\right)}\right)
\end{aligned}
$$

The equilibrium equation for cylindrical pressure vessel under the effect uniform magnetic field:

$$
\frac{d \sigma_{r}}{d r}+\frac{\sigma_{r}-\sigma_{\theta}}{r}+f_{z}=0
$$

where $f_{z}$ is the Lorentz's force derived from electrodynamic Maxwell equations [21] and defined as

$$
f_{z}=\mu(r)(\vec{J} \times \vec{H})=\mu_{i} H_{z}^{2}\left(e^{\omega(r-a)} \frac{\partial u}{\partial r}+e^{w(r-a)} \frac{u}{r}\right.
$$

Here $\sigma_{r}, \sigma_{\theta}, \varepsilon_{r}, \varepsilon_{\theta}, \vec{H}, \vec{J}, u$ correspond to radial and circumferential stresses, radial and circumferential strain, magnetic intensity vector, electric current density vector and displacement in radial direction, respectively. Substituting Eqs. (3.1) and material properties (2.1) into the governing equation (3.2), yields the following linear ordinary differential equation in terms of displacement:

$$
u^{\prime \prime}+Q_{c}(r) u^{\prime}+R_{c}(r) u=0
$$

where

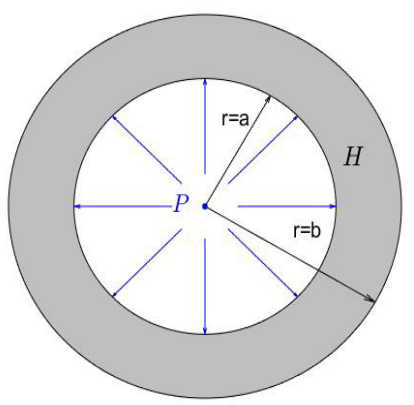

Figure 1. The cross-section of the FG pressure vessel subjected to internal pressure $(P)$ and uniform magnetic field $(H)$

$$
\begin{gathered}
Q_{c}(r)=\frac{\beta E(r)(1-v(r)) v_{1}(r)+2 \gamma^{2} E(r) v(r)^{2}(2-v(r))+H_{z}^{2} \mu(r) \omega v_{1}(r)^{2}}{E(r)(1-v(r)) v_{1}(r)+H_{z}^{2} \omega(r) v_{1}(r)^{2}}+\frac{1}{r} \\
R_{c}(r)=\frac{1}{r} \frac{(\beta+\gamma) E(r) v(r) v_{1}(r)+\gamma E(r) v(r)^{2}(1+4 v(r))+H_{z}^{2} \mu(r) \omega v_{1}(r)^{2}}{E(r)(1-v(r)) v_{1}(r)+H_{z}^{2} \mu(r) v_{1}(r)^{2}}-\frac{1^{2}}{r}
\end{gathered}
$$

with, $\quad v_{1}(r)=(1+v(r))(1-2 v(r))$. The boundary conditions in terms of displacement, derived from radial stress-strain relation (3.1a) and boundary conditions (2.3), can be written as

$$
\left[C_{11}(r) u^{\prime}+C_{12}(r) \frac{u}{r}\right]_{r=a}=-P
$$




$$
\left[C_{1} 1(r) u^{\prime}+C_{1} 2(r) \frac{u}{r}\right]_{r=b}=0
$$

\section{SPHERICAL VESSELS}

Functionally graded hollow sphere made from a mixture of metal and ceramic under the effect of a uniform magnetic field $\vec{H}\left(0,0, H_{\phi}\right)$ is considered. The stress-strain equations in spherical coordinate $(r, \theta, \varphi)$ can be written as follow:

$$
\begin{aligned}
& \sigma_{r}=C_{11}(r) \varepsilon_{r}+2 C_{12}(r) \varepsilon_{\theta} \\
& \sigma_{\theta}=\left[C_{11}(r)+C_{12}(r)\right] \varepsilon_{\theta}+C_{12}(r) \varepsilon_{r}
\end{aligned}
$$

The equilibrium equation for spherical pressure vessel under the effect uniform magnetic field:

$$
\frac{d \sigma_{r}}{d r}+\frac{2\left(\sigma_{r}-\sigma_{\theta}\right)}{r}+f_{\phi}=0
$$

where $f_{\phi}$ is the Lorentz's force derived from electrodynamic Maxwell equations [21] and defined as

$$
f_{\phi}=\mu(r)(\vec{J} \times \vec{H})=\mu_{i} H_{\phi}^{2}\left(e^{\omega(r-a)} \frac{\partial u}{\partial r}+e^{\omega(r-a)} \frac{2 u}{r}\right)
$$

Substituting Eqs. (4.1), (3.1c) and (4.3) into the governing equation (4.2), yields the following linear ordinary differential equation in terms of displacement:

$$
u^{\prime \prime}+Q_{s}(r) u^{\prime}+R_{s}(r) u=0
$$

Where

$$
\begin{aligned}
& Q_{s}(r)=\frac{\beta E(r)(1-v(r)) v_{1}(r)+2 \gamma^{2} E(r) v(r)^{2}(2-v(r))+H_{z}^{2} \mu(r) \omega v_{1}(r)^{2}}{E(r)(1-v(r)) v_{1}(r)+H_{z}^{2} \mu(r) v_{1}(r)^{2}}+\frac{2}{r} \\
& R_{s}(r)=\frac{1}{r} \frac{(\beta+\gamma) E(r) v(r) v_{1}(r)+\gamma E(r) v(r)^{2}(1+4 v(r))+H_{z}^{2} \mu(r) \omega v_{1}(r)^{2}}{E(r)(1-v(r)) v_{1}(r)+H_{z}^{2} \mu(r) v_{1}(r)^{2}}-\frac{2}{r^{2}}
\end{aligned}
$$

The boundary conditions in terms of displacement, derived from radial stress-strain relation (3.1a) and boundary conditions (2.3), can be written as

$$
\begin{aligned}
& {\left[C_{11}(r) u^{\prime}+2 C_{12}(r) \frac{u}{r}\right]_{r=a}=-P} \\
& {\left[C_{11}(r) u^{\prime}+2 C_{12}(r) \frac{u}{r}\right]_{r=b}=0}
\end{aligned}
$$

\section{SOLUTION PROCEDURE}

The solution of these differential equations ((3.4), (4.4)) are handled by both the Complementary Function Method (CFM) and the Pseudospectral Chebyshev Method (PCM). The former is solved the boundary value problem (BVP) in two steps: first the BVP is converted to system of initial value problem (IVP) and then solved any convenient method. The accuracy of this method is depend on the method used to solve IVP. For small mesh size it gives good results. The latter method only needs a good decompositions method for solving linear algebraic system. Although it results in spectral accuracy, in the need of big mesh size the accuracy slows down due the full differential matrix.

\subsection{Complementary Functions Method (CFM)}

The CFM is a method that allows the boundary value problem to be solved by converting it into an easily solvable system of initial value problem Fourth order Runge-Kutta method (RK4) is utilized to solve the system of initial value problems of concern. The detailed solution procedure of the CFM can be followed from the study of Tutuncu and Temel $[23,24]$.

The second order boundary value problem (3.4) can simply be converted by a simple change of variables $y_{1}=u_{c}$ and $y_{2}=u_{c}{ }^{\prime}$ to a system of two first order ordinary differential equation:

$$
Y^{\prime}=R h s\left(r, y_{1}, y_{2}\right)
$$

where

$$
Y=\left[\begin{array}{l}
y_{1} \\
y_{2}
\end{array}\right], R h s\left(r, y_{1}, y_{2}\right)=\left[\begin{array}{c}
y_{2} \\
\left(Q_{c}(r) y_{2}-R_{c}(r) y_{1}\right)
\end{array}\right]
$$

This system (5.1.1) is solved by selecting any dummy initial condition to ensure linear independence. Assuming that the complete solution of the second order boundary value problem is as follows:

$$
u_{c}=b_{j} u_{c j}, \quad j=1,2
$$

the coefficients $b_{j}, j=1,2$ are calculated from the boundary conditions (3.5). Note that by using CFM both the solution $\left(u_{c}(r)\right)$ and its derivative $\left(u_{c}(r)^{\prime}\right)$ are calculated at the same time.
The same solution procedure is followed for the solution of the spherical vessel.

\subsection{Pseudospectral Chebyshev Method (PCM)}

The Pseudospectral Chebyshev method [25,26] is based on the differential matrix approach and converts the differential equation into a system of linear equations. After that, the linear system can be solved by any decomposition method to achieve the desired accuracy.

The PCM is based on Chebyshev polynomials of the first kind, see, e.g., $[25,26]$. The first order $(N+1) \times(N+1)$ Chebyshev differentiation matrix associated with the Chebyshev-Gauss-Lobatto collocation points

$$
\begin{aligned}
& 0=r_{0}<r_{1} \ldots<r_{N}, \text { with } \\
& r_{j}=\cos (j \pi / N), j=0,1, \ldots, N
\end{aligned}
$$

will be denoted by $D$. First-order Chebyshev differentiation matrix $(D)$ provides highly accurate approximation to $u^{\prime}\left(r_{j}\right), u^{\prime \prime}\left(r_{j}\right), \ldots$, simply by multiplicating differential matrix with corresponding data vector $u^{\prime}\left(r_{j}\right)=(D u)_{j}, u^{\prime \prime}\left(r_{j}\right)=\left(D^{2} u\right)_{j}$, suchlike that $u=\left[u_{0}, \ldots, u_{n}\right]^{T}$ discrete vector data at positions $r_{j}$.

The computation procedure of the Chebyshev differentiation matrix and codes as an $\mathrm{m}$-file can be found in a notable references, see, e.g., Trefethen [26], where the collocation 
points $r_{j}$ are numbered from right to left and defined in $[-1,1]$. With a small adaptation, the $\mathrm{m}$-file of the differentiation matrix $D$ can be transcribed to any desired range $[a, b]$.

Efficiency, accuracy and the ease of implementation of the method are explained in detail in the study of Trefethen [26]. Therefore, the second order boundary value problem (3.4) is simply converted into a linear system by using the PCM as follows:

$$
\mathrm{M} u=0
$$

where

$$
M=D^{2}+Q_{s}(r) D+R_{s}(r)
$$

After imposing the boundary conditions (3.5) to this linear system (5.2.1), non-trival solution is obtained for the cylindrical body.

The same solution procedure is followed for the solution of the spherical vessel.

\section{RESULTS AND DISCUSSIONS}

The stress analysis of functionally graded thick hollow cylindrical and spherical vessels subjected to an internal pressure $P=50 \mathrm{MPa}$ under the effect of uniform magnetic field are numerically handled by both the Complementary Functions Method and the Pseudospectral Chebyshev Method. Inner and outer radius of the hollow bodies are $a=0.6, b=1$ respectively. It is assumed that the all material properties of the vessels and magnetic permeability are graded exponentially in radial direction. Two different mixture of ceramic and metal are used as a special material and the properties are given in Table 1 . The corresponding inhomogeneity parameters for the two mixture are calculated from Eqs. (2.2) and monitored in Table 2. It is obvious that selecting the parameters $\beta=\gamma=\omega=0$ results in homogeneous material that is pure metal. In this article, $T_{i}-6 \mathrm{Al}-4 \mathrm{~V}$ and $\mathrm{ZrO}_{2}$ will be expressed as the first material pair and Mullite and Molybdenum as the second material pair.

Table 1. Properties of Mixture (Ceramic-Metal)

\begin{tabular}{|c|c|c|}
\hline Material & $E(G P a)$ & $v$ \\
\hline $\mathrm{ZrO}_{2}$ (Ceramic) & 151 & $1 / 3$ \\
\hline $\mathrm{T}_{i}-6 \mathrm{Al}-4 \mathrm{~V}$ (Metal) & 116.7 & $1 / 3$ \\
\hline Mullite (Ceramic) & 225 & 0.27 \\
\hline Molybdenum (Metal) & 330 & 0.30 \\
\hline
\end{tabular}

Firstly, grid refinement test is made to compare the methods and show the accuracy as the mesh size increase. The solutions of a simple power-law grading function for only elastic modulus and magnetic permeability with fixed inhomogeneity parameters on the study of Dai et.al. [21] are used. The results in the midpoint of thickness for are presented with PCM and CFM in Table 3 for cylindrical vessel and Table 4 for spherical vessels.

Table 2. Inhomogeneity parameters for the two mixture

\begin{tabular}{|c|c|}
\hline$\beta$ & $\gamma$ \\
\hline 0.6442 & 0 \\
\hline-0.9575 & -0.2634 \\
\hline
\end{tabular}

Table 3. Grid refinement test of displacement in the midpoint of thickness ( $r=0.8)$ for FG cylindrical pressure vessel graded with a simple powerlaw

\begin{tabular}{|c|c|c|c|}
\hline $\mathrm{N}$ & PCM & CFM & Analytic [21] \\
\hline 4 & 0.888395290 & 0.888167625 & 0.888183995 \\
6 & 0.888192802 & 0.888181260 & 0.888183995 \\
8 & 0.888184239 & 0.888183197 & 0.888183995 \\
10 & 0.888184001 & 0.888183685 & 0.888183995 \\
12 & 0.888183995 & 0.888183851 & 0.888183995 \\
14 & 0.888183995 & 0.888183919 & 0.888183995 \\
16 & 0.888183995 & 0.888183952 & 0.888183995 \\
18 & 0.888183995 & 0.888183968 & 0.888183995 \\
20 & 0.888183995 & 0.888183978 & 0.888183995 \\
\hline
\end{tabular}

In order to compare of PCM and CFM, Chebyshev-Gauss-Lobatto collocation points, which are dense in the boundary and coarse on the other part, are used in the analysis. MATLAB software are used in all calculation. It can be noticed form the Tables 3-4 that the results obtained with PCM are better than the CFM. Furthermore, PCM results are in a good agreement with analytical solution and have at least six-digit accuracy by picking only 11 collocation points (10 intervals). Therefore, 11 collocation points are used after this stage in all the other calculation made in this manuscript. Additionally, the same analytic solutions of the displacement are used to compare the PCM and CFM along the thickness for 11 collocation points. It can be noticed form the Tables 5-6 that the results obtained along the thickness with PCM are better than the CFM.

Table 4. Grid refinement test of displacement in the midpoint of thickness $(r=0.8)$ for FG spherical pressure vessel graded with a simple powerlaw

\begin{tabular}{|c|c|c|c|}
\hline $\mathrm{N}$ & PCM & CFM & Analytic [21] \\
\hline 4 & 0.737408033 & 0.739588723 & 0.739206804 \\
6 & 0.739161920 & 0.739274518 & 0.739206804 \\
8 & 0.739205773 & 0.739227029 & 0.739206804 \\
10 & 0.739206781 & 0.739214779 & 0.739206804 \\
12 & 0.739206804 & 0.739210548 & 0.739206804 \\
14 & 0.739206804 & 0.739208785 & 0.739206804 \\
16 & 0.739206804 & 0.739207948 & 0.739206804 \\
18 & 0.739206804 & 0.739207509 & 0.739206804 \\
20 & 0.739206804 & 0.739207262 & 0.739206804 \\
\hline
\end{tabular}

Table 5. Comparison of displacement through the thickness for functionally graded cylindrical pressure vessel graded with a simple power law

\begin{tabular}{|c|c|c|c|}
\hline$r$ & PCM & CFM & Analytic [21] \\
\hline 0.6000 & 1.000000000 & 1.000000000 & 1.000000000 \\
\hline 0.6098 & 0.994015053 & 0.994015046 & 0.994015053 \\
\hline 0.6382 & 0.976911255 & 0.976911216 & 0.976911253 \\
\hline 0.6824 & 0.951136104 & 0.951135972 & 0.951136101 \\
\hline 0.7382 & 0.920267918 & 0.920267665 & 0.920267913 \\
\hline 0.8000 & 0.888184001 & 0.888183685 & 0.888183995 \\
\hline 0.8618 & 0.858274209 & 0.858273890 & 0.858274202 \\
\hline 0.9176 & 0.833049965 & 0.833049661 & 0.833049957 \\
\hline 0.9618 & 0.814141670 & 0.814141375 & 0.814141662 \\
\hline 0.9902 & 0.802490047 & 0.802489757 & 0.802490039 \\
\hline 1.0000 & 0.798560219 & 0.798559930 & 0.798560211 \\
\hline
\end{tabular}

In the absence of a magnetic field, the displacement and stress distributions of the functionally graded cylinder and sphere are shown in Figure 2 for the first material pair and Figure 3 for the second material pair. Two different material models are compared with different homogeneous materials 


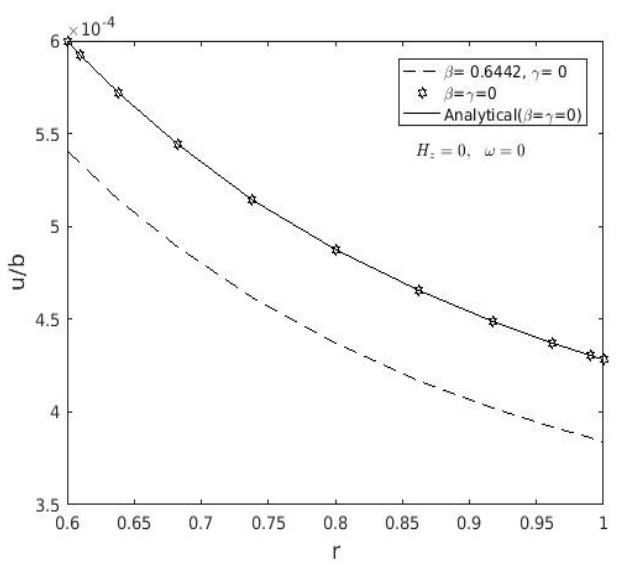

(a)

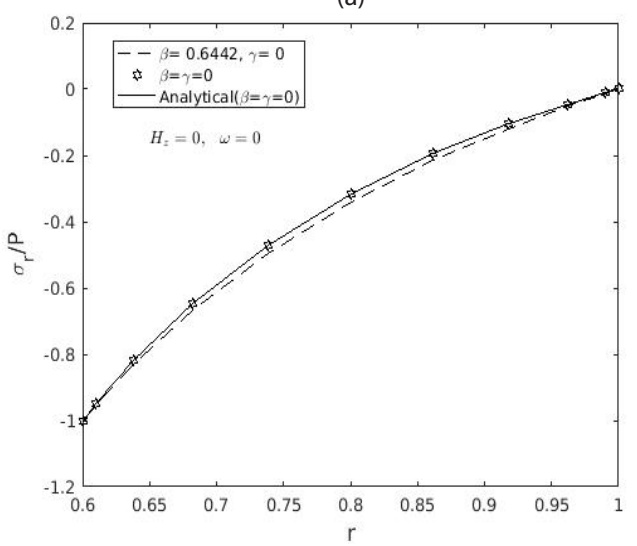

(c)

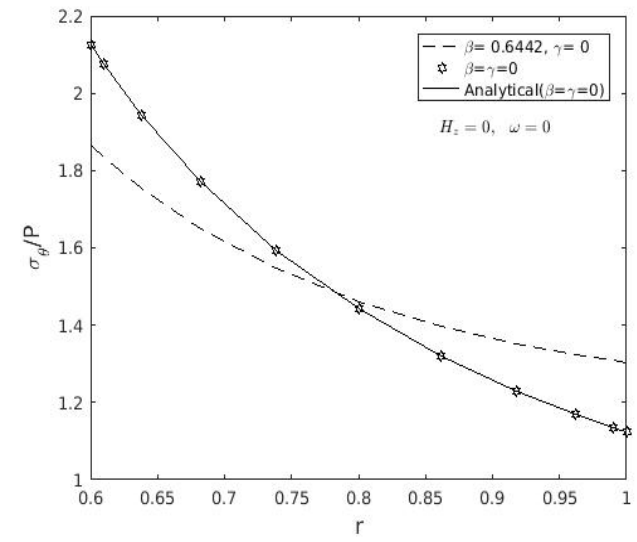

(e)

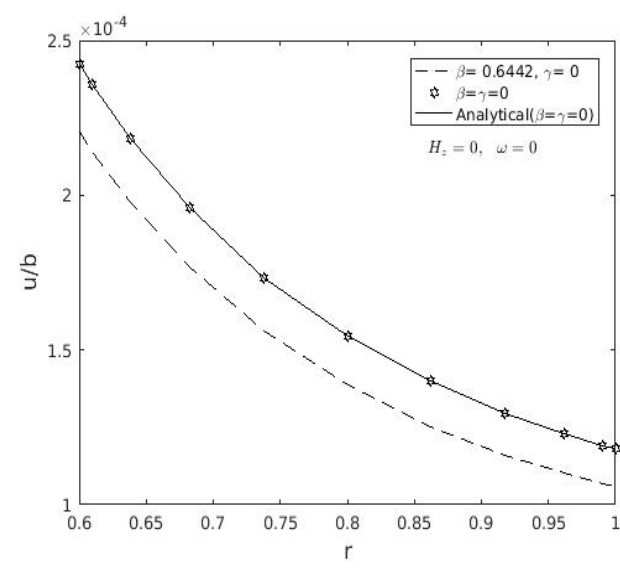

(b)

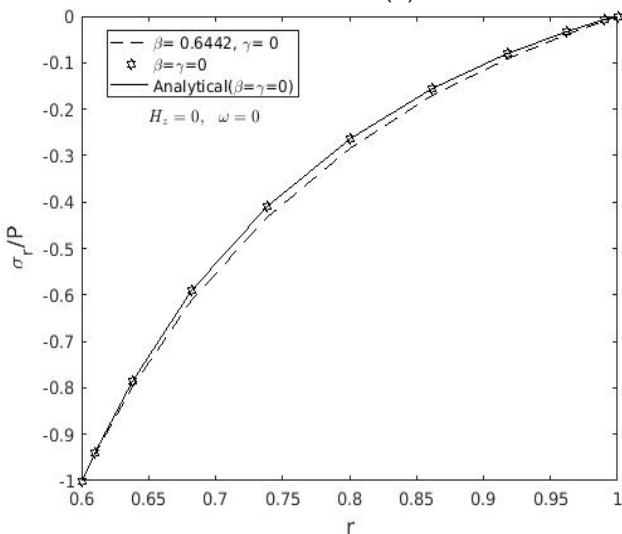

(d)

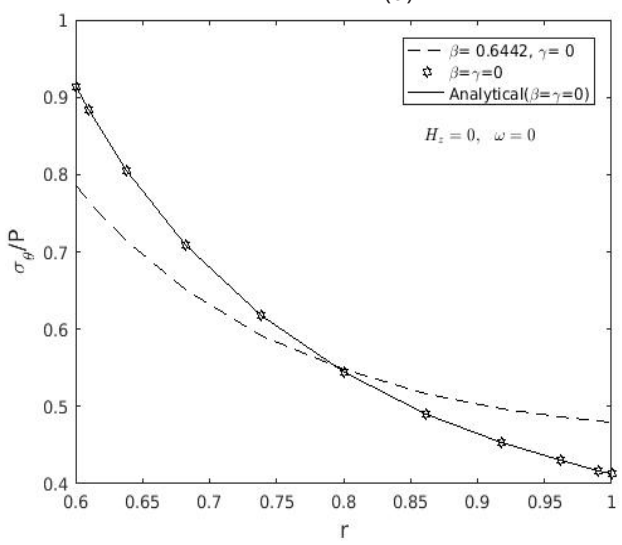

(f)

Figure 2. In the absence of magnetic field, the comparison of the functionally graded cylindrical and spherical vessels with the homogeneous material for the first material pair (Poisson ratio constant). The figures in the left column are corresponding to the hollow cylinder and the right column to hollow sphere

(dashed line). In the first mixture the homogeneous material corresponds to the metal $\mathrm{Ti}-6 \mathrm{Al}-4 \mathrm{~V}$, while in the second mixture the homogeneous material corresponds to the metal molybdenum. According to the properties given in Table 1 , the elastic modulus of the metal in the first material pair is smaller than the second material pair. While the Poisson's ratio is taken constant in the first mixture, both elastic modulus and Poisson's ratio are taken variable in the second mixture. The displacements occurring in both the functionally graded cylindrical and spherical pressure vessels obtained from the first material mixture are lower than in the homogeneous material state (Figure 2(a-b)), while they are higher in the second material mixture (Figure $3(\mathrm{a}-\mathrm{b})$ ). It is observed that the radial stress occurs in the compression direction (Figure 2-3(c-d)), while the circumferential stress

Table 6. Comparison of displacement through the thickness for functionally graded spherical pressure vessel graded with a simple power law

\begin{tabular}{|c|c|c|c|}
\hline$r$ & PCM & CFM & Analytic [21] \\
\hline 0.6000 & 1.000000000 & 1.000000000 & 1.000000000 \\
\hline 0.6098 & 0.982784793 & 0.982785091 & 0.982784795 \\
\hline 0.6382 & 0.936154077 & 0.936155292 & 0.936154084 \\
\hline 0.6824 & 0.872067323 & 0.872070469 & 0.872067336 \\
\hline 0.7382 & 0.803290365 & 0.803296210 & 0.803290384 \\
\hline 0.8000 & 0.739206781 & 0.739214779 & 0.739206804 \\
\hline 0.8618 & 0.685015182 & 0.685024070 & 0.685015208 \\
\hline 0.9176 & 0.642822554 & 0.642831428 & 0.642822582 \\
\hline 0.9618 & 0.613039354 & 0.613047930 & 0.613039383 \\
\hline 0.9902 & 0.595403517 & 0.595411856 & 0.595403546 \\
\hline 1.0000 & 0.589572052 & 0.589580310 & 0.589572082 \\
\hline
\end{tabular}




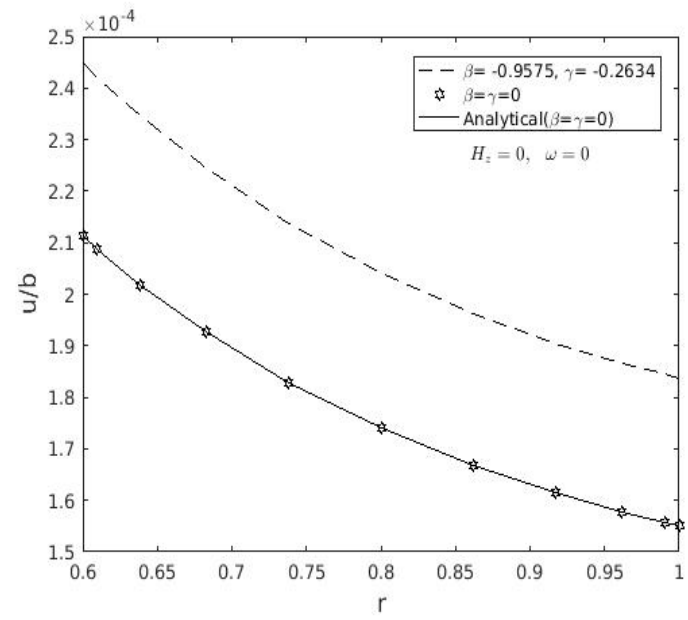

(a)

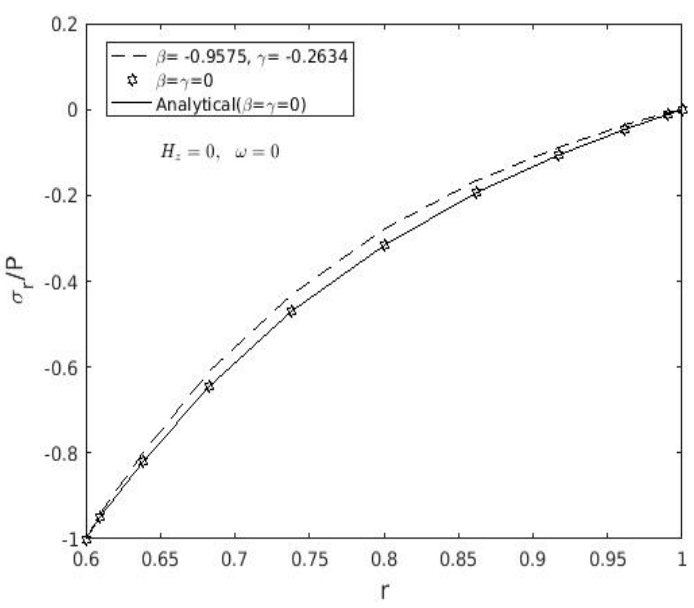

(c)

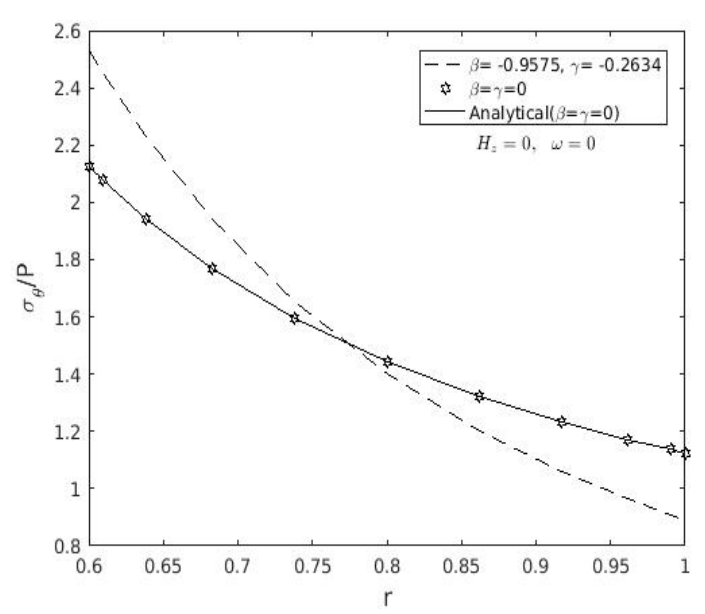

(e)

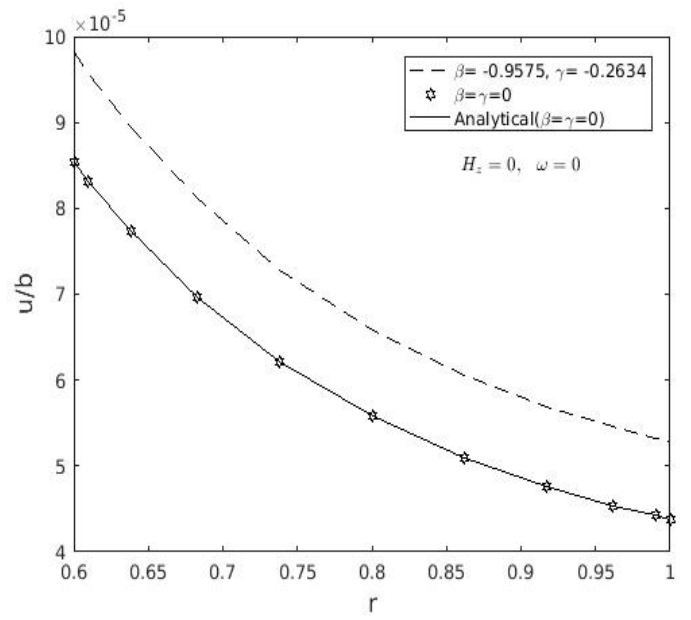

(b)

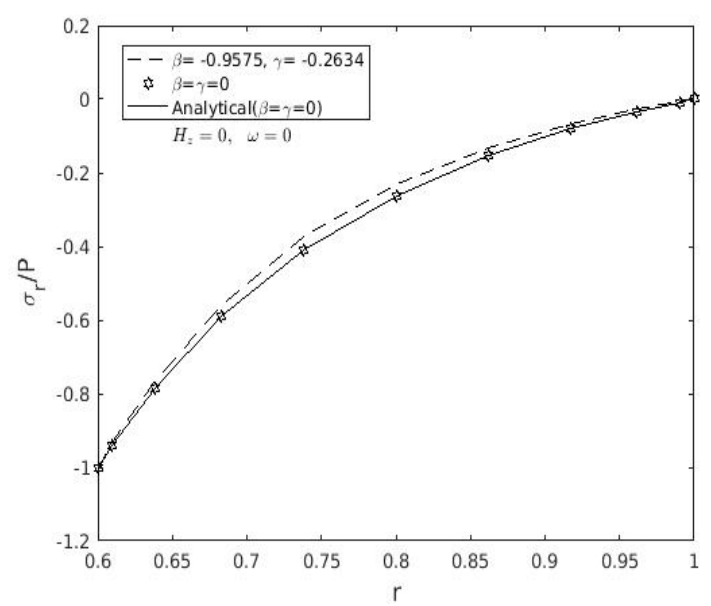

(d)

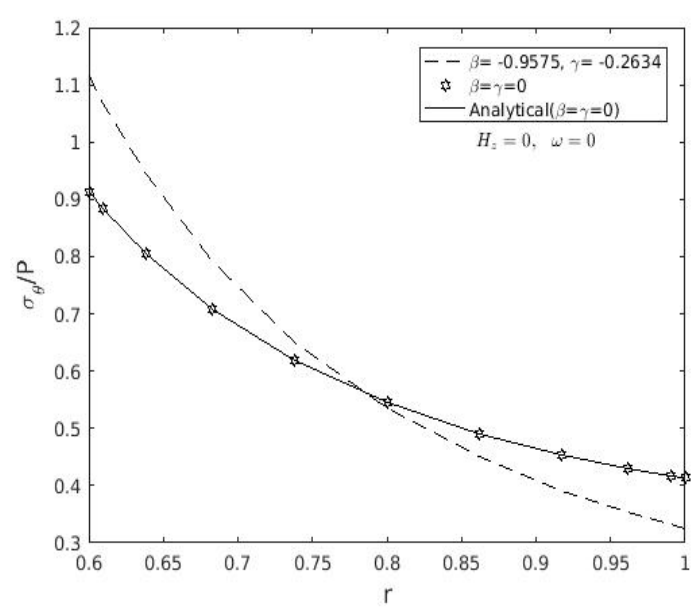

(f)

Figure 3. In the absence of magnetic field, the comparison of the functionally graded cylindrical and spherical vessels with the homogeneous material for the second material pair. The figures in the left column are corresponding to the hollow cylinder and the right column to hollow sphere

occurs in the tensile direction (Figure 2-3(e-f)). In the second pair of materials, the radial stress remains relatively low than the homogeneous one (Figure 3(c-d)). For the first pair of materials, the circumferential stress of the graded material of both geometries is lower than the homogeneous one in the inner wall, but higher in the outer wall (Figure 2(e-f)). The opposite is true for the second material pairs (Figure $3(\mathrm{e}-\mathrm{f}))$. In addition, it is seen that the analytical results obtained for the homogeneous material coincide with the numerical results in all conditions.

The displacement, radial and circumferential stress results are given for the different permeability coefficients under the influence of internal pressure and uniform magnetic field in cylindrical and spherical geometry for two different material pairs (Figure 4). The solid line indicates the first material 


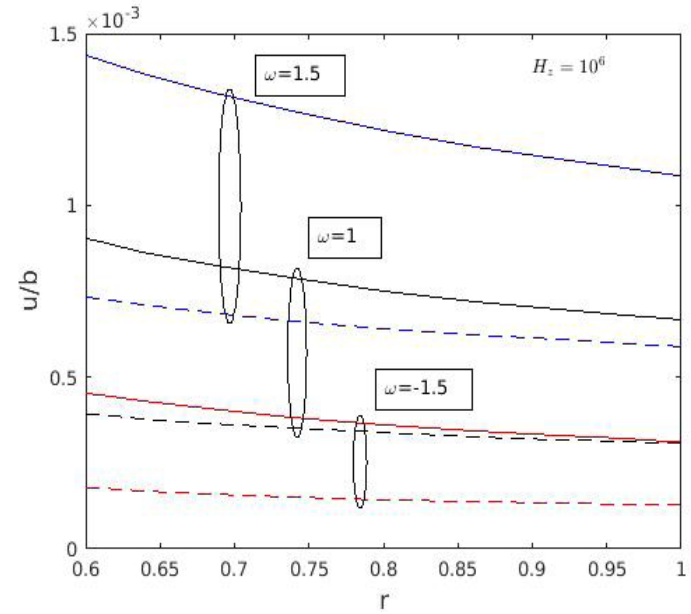

(a)

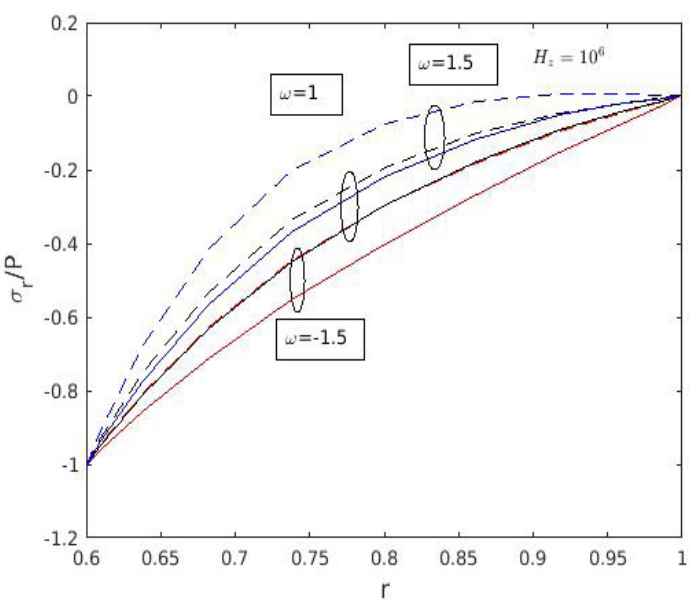

(c)

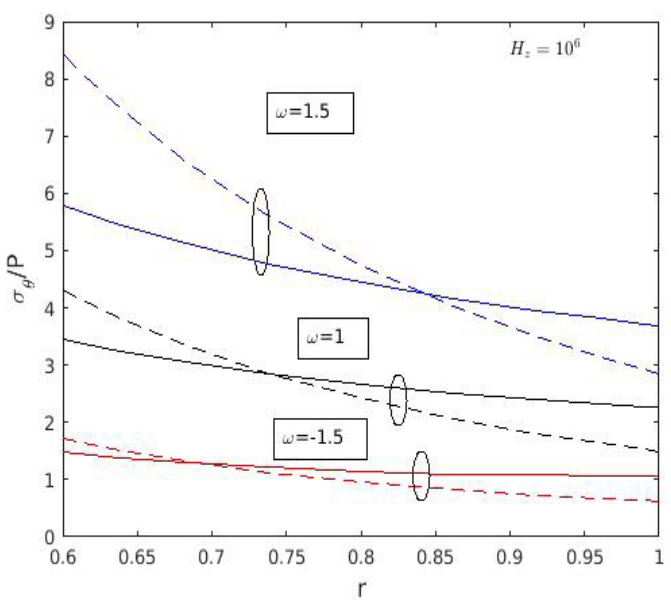

(e)

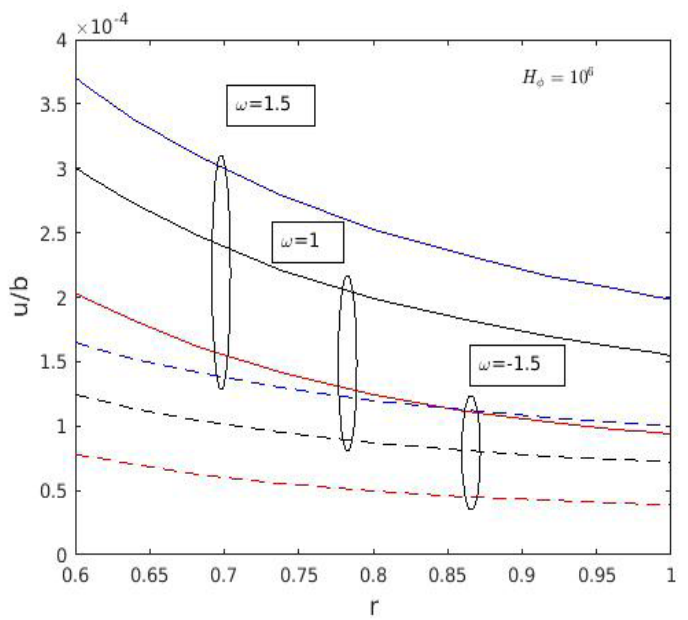

(b)

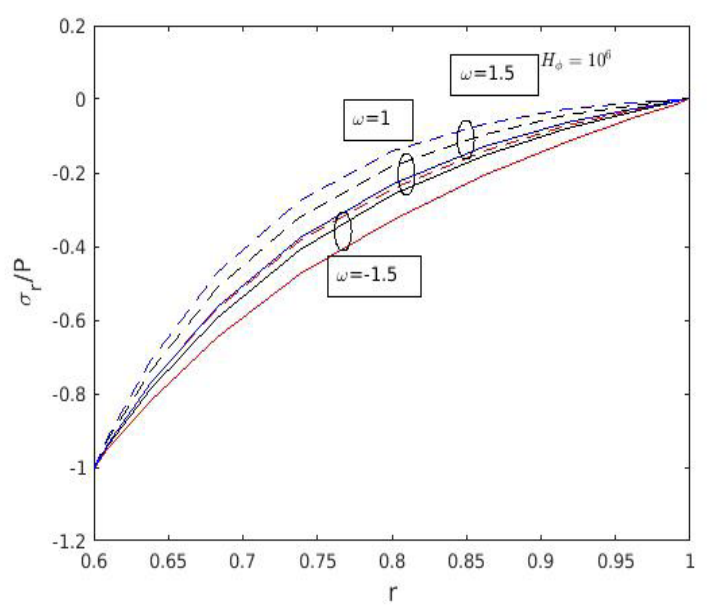

(d)

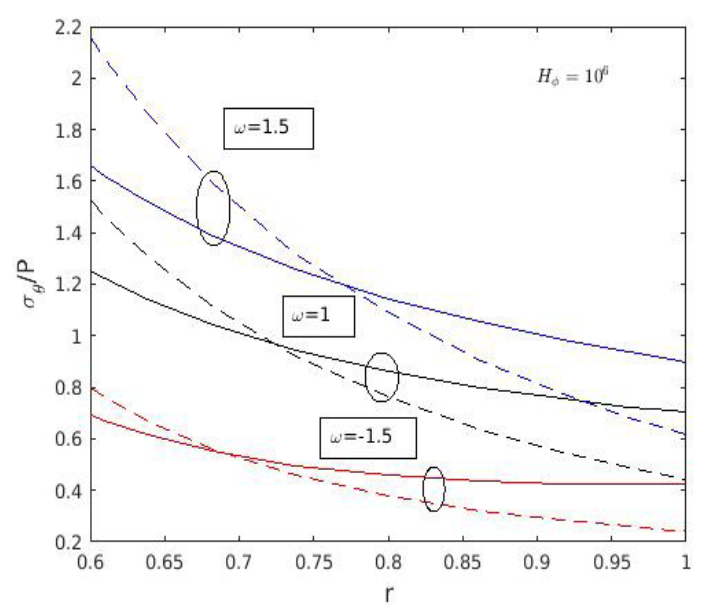

(f)

Figure 4. The effect of magnetic permeability on displacement and stresses in a functionally graded cylindrical and spherical vessels under the effect of uniform magnetic field. First material pair is shown with solid line and second material pair with dashed line. The figures in the left column are corresponding to the hollow cylinder and the right column to hollow sphere.

pair, while the dashed line indicates the second material pair. The ellipses on the figures are used to show two material pairs for different permeability coefficients. In Figure 4(a-b), it is seen that the displacements reach larger values in the inner wall for both geometries. The displacements increase with increasing permeability coefficient, which is more obvious for the first material pair. In the second pair of ma- terials, minus the permeability coefficient ensures that the displacements remain both lower and similar along the wall. For all geometries and conditions, the radial stress occurs in the compression direction (Figure $4(\mathrm{c}-\mathrm{d})$ ). The negative magnetic permeability coefficient keeps radial stresses at low values, but its effect is not as determinative as in displacements. It is observed that spherical geometries are not sig- 
nificantly affected by the change in permeability coefficient (Figure 4(d)). The amount of circumferential stress occurring in tensile direction from the inner wall to the outer wall in the second pair of materials is higher than the first pair of materials (Figure 4(e-f)). For example, for the second material pair $(\mathrm{w}=1.5)$, the circumferential tensile strength ratio between the inner wall and the outer wall in the cylinder geometry is greater than twice, whereas in the first material pair this difference is less than 1.5 times. This is proportional to the magnitude of the elastic modulus values of the material pairs. When the stress distributions are examined, it can be concluded that the permeability coefficient is more effective in controlling circumferential stress as opposed to radial stress. In order to keep the circumferential stress distribution along the wall at the lowest possible value and close to the constant, it would be more appropriate to prefer the first pair of materials with minus permeability coefficient in both geometries.

\section{CONCLUSIONS}

In this research, the stress analysis of functionally graded thick hollow cylindrical and spherical vessels subjected to an internal pressure under the effect of uniform magnetic field are investigated. It is assumed that the all material properties of the hollow bodies are graded exponentially in radial direction. These conditions produce a linear ordinary differential equation. These differential equations are numerically handled by both the Complementary Function Method and the Pseudospectral Chebyshev Method. Firstly, grid refinement test is made to compare the methods and show the accuracy as the mesh size increase. The solutions of a simple power-law grading function for only elastic modulus and magnetic permeability with fixed inhomogeneity parameters on the study of Dai et.al. [21] are used. It can be noticed that PCM are better than the CFM and have at least six-digit accuracy by picking only 11 collocation points (10 intervals). Additionally, the same analytic solutions of the displacement are used to compare the PCM and CFM along the thickness for 11 collocation points. It can be noticed from the Tables 5-6 that the results obtained along the thickness with PCM are better than the CFM. Primarily, the effect of two different ceramic-metal mixtures on displacement and stresses is investigated in the absence of magnetic field. It has been shown that displacement and stress values can be reduced by appropriate selection of the material. Then, the displacement and stress values under the effect of uniform magnetic field for different magnetic permeability values are investigated in two materials simultaneously. The effect of magnetic field and permeability on displacement and stresses is discussed. It can be concluded that FG materials have strong effect on the stresses and displacement, and magnetic field can be used to optimize the structural performance.

\section{Acknowledgement}

The study was supported by OKÜBAP-2019-PT3-013.

\section{REFERENCES}

[1] Yamanouchi, M., Koizumi, M., Hirai, T. and Shiota, I. (1990). FGM '90. Proceedings of the 1st International Symposium on Functionally Gradient Materials. 8-9 October, Sendai.

[2] Koizumi, M. (1993). The concept of FGM, Ceramic Transactions, Functionally Gradient Materials, 34:3-10.

[3] Erdogan, F. (1995). Fracture mechanics of functionally graded materials. Composite Engineering. 5(7):753-770, DOI:10.1016/09619526(95)00029-M

[4] Horgan, C.O., Chan, A.M., The pressurized hollow cylinder or disk problem for functionally graded isotropic linearly elastic materials, Journal of Elasticity, 55, 43-59, 1999, DOl:10.1023/A:1007625401963.

[5] Tutuncu, N. and Ozturk, M. (2001). Exact solutions for stresses in functionally graded pressure vessels. Composites: Part B, 32:683686, DOI:10.1016/S1359-8368(01)00041-5.

[6] Tutuncu, N. (2007). Stresses in thick-walled FGM cylinders with exponentially-varying properties. Engineering Structures, 29:20322035, DOI:10.1016/j.engstruct.2006.12.003.

[7] Yarımpabuc, D., Eker, M. and Celebi, K. (2018). Mechanical behavior of functionally graded pressure vessels under the effect of Poisson's ratio. European Mechanical Science, 2(2):52-59, DOI: 10.26701/ ems.360134.

[8] Eker, M., Yarımpabuc, D., Celebi, K., Yıldırım, A. (2017). Stress analysis of functionally graded cylindrical pressure vessels by pseudospectral chebyshev method. IAREC 2017 Conference Proceedings, p. 481.

[9] Eker M., Yarımpabu, c, D., Celebi K., Yıldırım, A. (2017). Stress analysis of functionally graded spherical pressure vessels by pseudospectral chebyshev method. IMSEC 2017 Conference Proceedings, p. 417.

[10] Chen, Y.Z. and Lin, X.Y. (2008). Elastic analysis for thick cylindrical and spherical pressure vessels made of functionally graded materials. Computational Material Science, 44(2):581-587, DOI:10.1016/j. commatsci.2008.04.018.

[11] Li, X.F., Peng, X.L. and Kang, Y.A. (2009). Pressurized hollow spherical vessels with arbitrary radial nonhomogeneity. AIAA Journal, 47(9):2263-2265, DOI:10.2514/1.41995.

[12] Nejad, M.Z. and Gharibi, M. (2014). effect of material gradient on stresses of thick fgm spherical pressure vessels with exponentially-varying properties. Journal of Advanced Materials and Processing, 2(3):39-46.

[13] Temel, B, Yildirim S, Tutuncu N. (2014). Elastic and viscoelastic response of heterogeneous annular structures under Arbitrary Transient Pressure. International Journal of Mechanical Sciences, 89:78-83, DOI: 10.1016/j.jimecsci.2014.08.021

[14] Baş, H., Keles I. (2015). Novel approach to transient thermal stress in an annular fin. Journal of Thermophysics and Heat Transfer, 29(4), 705-710, DOI:10.2514/1.T4535.

[15] Baba, S., Keles I. (2016). A novel approach to forced vibration behavior of thick-walled cylinders. International Journal of Pressure Vessels and Piping, 137, 22-27, DOI:10.1016/j.jpvp.2015.04.015.

[16] Keles, I. (2016). Novel approach to forced vibration behavior of anisotropic thick-walled spheres. AIAA Journal, 54(4), 1438-1442, DOI:10.2514/1.J054322.

[17] Temel, B., Aslan, T.A., and Noori, A.R., (2017). An efficient dynamic analysis of planar arches. European Mechanical Science, 1(3), 82-88, DOI:10.26701/ems.325808.

[18] Arslan, E. (2017). Tabakalı ve fonksiyonel olarak kademelendirilmis, küresel basınç kapları üzerine analiz. Pamukkale Üniversi- 
tesi Mühendislik Bilimleri Dergisi, 23(1), 24-35, DOl:10.5505/pajes.2016.56688.

[19] Noori, A.R., Aslan T.A., Temel, B. (2018). An efficient approach for in-plane free and forced vibrations of axially functionally graded parabolic arches with nonuniform cross section. Composite Structure, 200:701-710, DOI:10.1016/j.compstruct.2018.05.077.

[20] Yildirim, S. and Tutuncu, N. (2019). Effect of magneto-thermal loads on the rotational instability of heterogeneous rotors, AIAA Journal, 57(5):2069-2074, DOI:10.2514/1.J058124.

[21] Dai, H.L., Fu, Y.M. and Dong, Z.M. (2006). Exact solutions for functionally graded pressure vessels in a uniform magnetic field. International Journal of Solids and Structures, 43:5570-5580, DOl:10.1016/j. ijsolstr.2005.08.019.

[22] Dai, H.L., Xiao, X. and Fu, Y.M. (2010). Analytical solutions of stresses in functionally graded piezoelectric hollow structures. Solid State Communications, 150:763-767, DOI:10.1016/j.ssc.2010.01.028.

[23] Tutuncu, N. and Temel, B. (2009). A novel approach to stress analysis of pressurized FGM cylinders, disks and spheres. Composite Structure, 91(3):385-390, DOl:10.1016/j.compstruct.2009.06.009.

[24] Tutuncu, N. and Temel, B. (2013). An efficient unified method for thermoelastic analysis of functionally graded rotating disks of variable thickness. Mechanics of Advanced Materials and Structures, 30(1):38-46, DOl: 10.1080/15376494.2011.581413.

[25] Gottlieb, D. (1981). The stability of pseudospectral-Chebyshev methods. Mathematics of Computation, 36(153):107-118, DOI:10.2307/2007729.

[26] Trefethen, L.N. (2000). Spectral methods in Matlab, SIAM, Philadelphia, PA. 